\title{
Improvement of NADPH bioavailability in Escherichia coli through the use of phosphofructokinase deficient strains
}

\author{
Yipeng Wang • Ka-Yiu San • George N. Bennett
}

Received: 6 November 2012 / Revised: 11 March 2013 / Accepted: 13 March 2013

(C) Springer-Verlag Berlin Heidelberg 2013

\begin{abstract}
NADPH-dependent reactions play important roles in production of industrially valuable compounds. In this study, we used phosphofructokinase (PFK)-deficient strains to direct fructose-6-phosphate to be oxidized through the pentose phosphate pathway (PPP) to increase NADPH generation. $p f k A$ or $p f k B$ single deletion and double-deletion strains were tested for their ability to produce lycopene. Since lycopene biosynthesis requires many NADPH, levels of lycopene were compared in a set of isogenic strains, with the $p f k A$ single deletion strain showing the highest lycopene yield. Using another NADPH-requiring process, a one-step reduction reaction of 2-chloroacrylate to 2-chloropropionic acid by 2haloacrylate reductase, the $p f k A p f k B$ double-deletion strain showed the highest yield of 2-chloropropionic acid product. The combined effect of glucose-6-phosphate dehydrogenase overexpression or lactate dehydrogenase deletion with PFK deficiency on NADPH bioavailability was also studied. The results indicated that the flux distribution of fructose-6phosphate between glycolysis and the pentose phosphate pathway determines the amount of NAPDH available for reductive biosynthesis.
\end{abstract}

Keywords PFK $\cdot$ NADPH bioavailability $\cdot p f k A \cdot p f k B$. $\mathrm{G} 6 \mathrm{PDH} \cdot$ E. coli

\footnotetext{
Y. Wang · G. N. Bennett $(\bowtie)$

Department of Biochemistry and Cell Biology, MS-140,

Rice University, 6100 Main Street,

Houston, TX 77005-1892, USA

e-mail: gbennett@rice.edu

K.-Y. San

Department of Bioengineering, Rice University, Houston, TX 77005, USA

K.-Y. San

Department of Chemical and Biomolecular Engineering, Rice University, Houston, TX 77251, USA
}

\section{Introduction}

Cofactor pairs, $\mathrm{NAD}^{+} / \mathrm{NADH}$ and $\mathrm{NADP}^{+} / \mathrm{NADPH}$ are essential for the growth and energetics of microbial cells. NADH molecules are oxidized mainly through the electron transport chain and serve as the source of ATP under aerobic conditions, while NADPH molecules are mainly oxidized through anabolic pathway and provide essential reducing cofactors for macromolecular biosynthesis such as fatty acids, amino acids, and nucleic acid components. Internal availability of $\mathrm{NADP}^{+} / \mathrm{NADPH}$ make microbial cells perfect candidates for whole cell catalyzed redox reactions for the production of industrially valued compounds. However, the relative composition of the reduced form of $\mathrm{NADP}^{+}$/ NADPH and the overall concentration inside the cells is tightly controlled and balanced with $\mathrm{NAD}^{+} / \mathrm{NADH}$ through pyridine nucleotide transhydrogenase and the biosynthetic enzymes involved in NAD and NADP synthesis. To utilize intracellular NADPH as the reducing power to drive redox reactions, different strategies have been developed and investigated in Escherichia coli to increase the bioavailability of NADPH.

The reduced cofactor, NADPH, is mainly generated through the pentose phosphate pathway (PPP), isocitrate synthase of the tricarboxylic acid cycle (TCA), and malic enzyme (Fig. 1). Strategies such as overexpression of $z w f$, encoding glucose-6-phosphate dehydrogenase (G6PDH) (Chin et al. 2009; Kim et al. 2011b; Lee et al. 2007), or deletion of pgi, encoding phosphoglucose isomerase (Bartek et al. 2010; Chemler et al. 2010; Kim et al. 2011a), have been used to increase the metabolic flux to PPP from glycolysis. Other strategies include overexpression of pyridine nucleotide transhydrogenase encoded by $u d h A$ which converts NADH to NADPH (Sanchez et al. 2006), and switching the glycolytic enzyme, $\mathrm{NAD}^{+}$dependent glyceraldehyde-3-phosphate dehydrogenase (GAPDH) to 
Fig. 1 Simplified diagram of glycolysis, TCA cycle, PPP, and biosynthesis pathway of lycopene. Abbreviations used are as follows: G6P glucose-6phosphate, F6P fructose-6phosphate, $G 3 P$ glyceraldehyse-3-phosphate, $P E P$ phosphoenolpyruvate, 6PG 6-phosphogluconolactone, $R U 5 P$ ribulose-5-phosphate, $R 5 P$ ribose-5-phosphate, $X 5 P$ xylulose-5-phosphate, $S 7 P$ sedoheptulose-7-phosphate, E4P erythrose-4-phosphate, DHAP dihydroxyactone-3phosphate, $D X P$ deoxyxylulose-5-phosphate, $D M P P$ dimethylallyl pyrophosphate, IPP isopentenyl phosphate

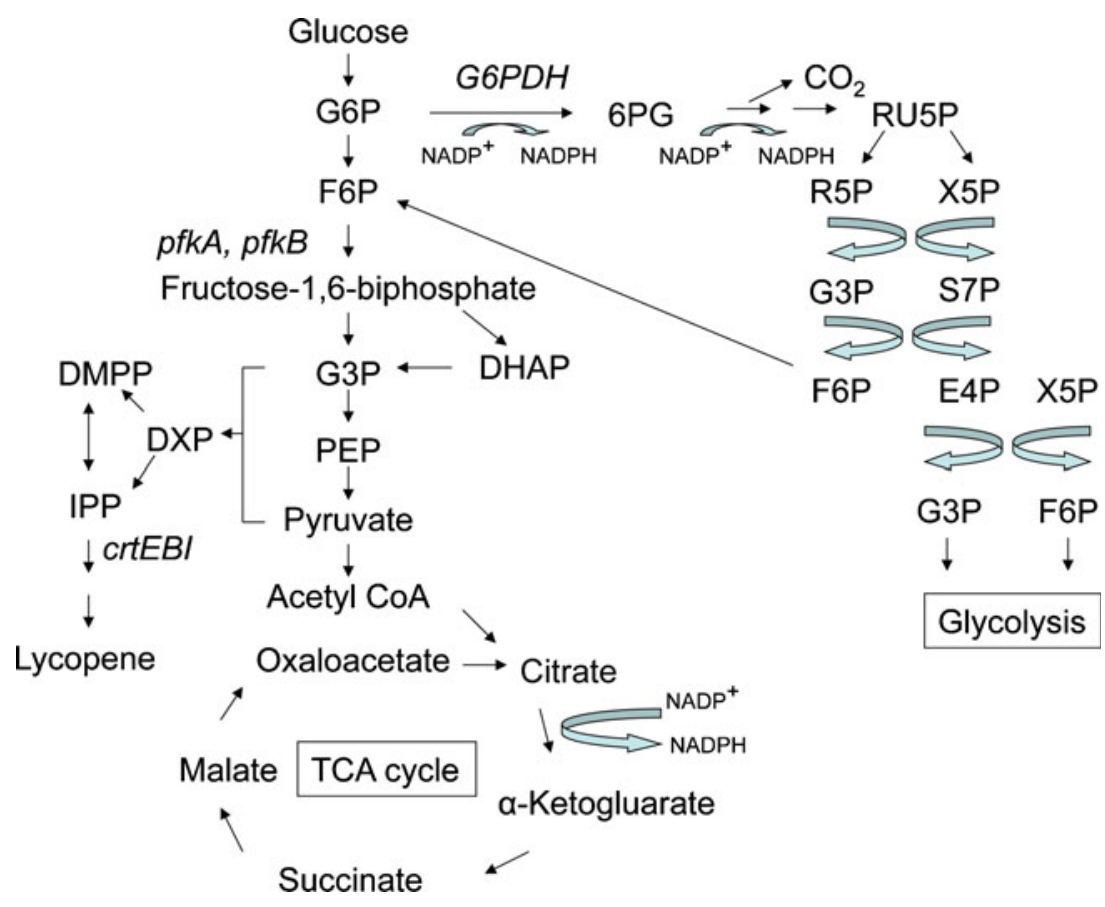

$\mathrm{NADP}^{+}$-dependent GAPDH from Clostridium acetobutylicum (Martinez et al. 2008).

In this study, we focused on phosphofructokinase which catalyzes the phosphorylation of fructose-6-phosphate to fructose-1,6-biphosphate and is the first committed step of the major glycolytic pathway in bacteria. There are two isoenzymes of PFK in E. coli, PfkA and PfkB. PfkA has been reported to contribute $90 \%$ of the PFK activity while PfkB is the minor enzyme contributing $5-10 \%$ of the activity (Vinopal et al. 1975). In another study (Siedler et al. 2011), pfkA mutants showed $23 \%$ of wild-type activity while $p f k B$ mutants showed $78 \%$ of wild-type activity, with the double mutant exhibiting $27 \%$ of wild-type activity. These two enzymes have different kinetic properties, e.g., PfkB is little affected by phosphoenolpyruvate levels and does not show cooperativity in response to fructose-6phosphate in contrast to PfkA. It is possible that these differences in enzyme properties contribute to variation in metabolism in individual mutant strains under certain conditions. Previous studies have shown that deleting $p f k A$ alone improved NADPH-dependent xylitol production from xylose (Chin and Cirino 2011). We are studying the effects of blocking this step entirely by deleting both PFK genes, $p f k A$ and $p f k B$, and consider that disrupting glycolysis at this point will then force fructose-6-phosphate to undergo the reversible isomerization reaction that proceeds in the direction of glucose 6-phosphate. Glucose 6-phosphate will then enter the PPP, which will generate $2 \mathrm{~mol}$ of NADPH per cycle. Normally, the fructose 6-phosphate resulting from the non-oxidative stage of PPP will enter glycolysis, but in the absence of both $p f k A$ and $p f k B$, theoretically, it will be converted to glucose 6-phophate and can proceed back to PPP again, which will lead to more NADPH generation. This has been recently described as a partial cyclization of the pentose phosphate pathway (Siedler et al. 2012). Glyceraldehyde 3-phosphate resulting from PPP will reenter the glycolytic pathway and TCA cycle in the end to generate NADH and ATP. By forcing fructose 6-phosphate to go through the PPP to be fully oxidized, the overall theoretical balance will be $1 \mathrm{~mol}$ of glucose input with $1 \mathrm{~mol}$ of glyceraldehyde 3-phosphate, $3 \mathrm{~mol}$ of $\mathrm{CO}_{2}$, and 6 mol of NADPH as output from the steps leading to the glycolytic intermediate, glyceraldehyde 3-phosphate. Thus, the maximal NADPH that could be derived from $1 \mathrm{~mol}$ of glucose through this PPP process would be the six derived from this PPP process plus those derived from GAP metabolism, e.g., another NADPH could be derived from the isocitrate dehydrogenase reaction in the TCA cycle. In addition, the presence of transhydrogenases may contribute some via the conversion of NADH from other reactions to NADPH.

We wanted to assess the effect of rerouting metabolism on NADPH availability in different circumstances that would represent situations where such modifications might be used and would allow the function to be determined in the context of cellular redox metabolism and with various products. The systems chosen for analysis of the effects were: lycopene as a representative of a highly NADPHdependent metabolite derived from glycolytic precursors formed in aerobic cultures and representative of situations where normal cellular precursors are channeled to a desired product; and in a controlled anaerobic biocatalyst mode 
where we could analyze the total redox of the cell going to reduced products and assess the contribution of the added exogenous NADPH-dependent system "sink" in competition with other redox processes in the cell (e.g., lactate formation).

We examined the PFK-deficient strains in situations where a product highly dependent on NADPH was being made. These situations included NADPH-dependent lycopene production and one-step reduction of 2-chloroacrylate to 2-chloropropionic acid, two compounds that we could accurately measure as indicators of NADPH use. Lycopene is a commercially important carotenoid that has antioxidant activity and other health benefits. Lycopene producing genes crtEBI from Erwinia herbicola have been expressed in $E$. coli and lycopene has been successfully synthesized in E. coli (Alper et al. 2005b; Yoon et al. 2008). The biosynthesis of lycopene in $E$. coli follows the non-mevalonate pathway that uses the glycolytic intermediate glyceraldehyde-3phosphate and pyruvate as precursors and would consume $16 \mathrm{~mol}$ of cofactor NADPH for $1 \mathrm{~mol}$ of lycopene production (Alper et al. 2005a).

The one-step reaction module we used to demonstrate NADPH bioavailability uses 2-haloacrylate reductase CAA43 from the soil bacteria, Burkholderia sp. WS (Kurata et al. 2008; Kurata et al. 2005). CAA43 catalyzes reduction of the carbon-carbon double bond of 2-chloroacrylate to (s)-2chloropropionate coupled with NADPH oxidization to $\mathrm{NADP}^{+}$. This reaction directly reflects the bioavailability of NADPH in the cells and by supplying enough substrate 2chloroacrylate, an ample sink will be provided for the cell to oxidize extra NADPH, and therefore to maintain redox homeostasis. This also provides an example for the more practical situation of industrial relevance, where a NADPHdependent product is desired to be formed at high levels.

This study showed that deletion of both PFK genes, $p f k A$ and $p f k B$, improved bioavailablity of NADPH in the cell as analyzed by the 2-chloropropionate production system. Lycopene production was the highest in the $p f k A$ single deletion strain. Both NADPH-dependent processes were also studied with overexpression of G6PDH to further increase the PPP flux and by deletion of lactate dehydrogenase $(l d h)$ which would prevent NADH consumption by formation of this fermentation byproduct.

\section{Materials and methods}

Bacterial strains and plasmids

Table 1 describes the strains and plasmids used in this study. For the lycopene production assay, E. coli MQ, RL257, RL254, and RL255 were transformed with pACLYC carrying carotenoid biosynthesis genes $c r t E$, $c r t I$, and $c r t B$ from E. herbicola. For 2-haloacrylate reductase assay, MQ, RL257,
RL254, and RL255 were transformed with pTrc99A-CAA43, respectively. G6PDH was overexpressed from plasmid pASK-IBA-G6PD1. In the case of the 2-haloacrylate reductase assay, to be compatible with pASK-IBA-G6PD1, the gene encoding the 2-haloacrylate reductase, CAA43 was moved from $\mathrm{pTr} 99 \mathrm{~A}-\mathrm{CAA} 43$ to $\mathrm{pDHC} 29$.

The gene encoding the 2-haloacrylate reductase, CAA43 was amplified by PCR from pET101-D-Topo-CAA43 using primers CAA43-F-NcoI (5'-CCG CCATGG GA ATG GTA ATG GCA GCG GTA ATT CAT AAG-3') and CAA43-RXbaI (5'-CGC TCTAGA CTA CGC TTG CGG AAG CAA AAC AAT-3'). The PCR fragment was digested with NcoI and $X b a \mathrm{I}$ and cloned into pTrc99A. To make pDHC29CAA43, CAA43 was amplified by PCR using primers CAA43-F-SacI (5'-CCG GAGCTC C ATG GTA ATG GCA GCG GTA ATT CAT AAG-3') and CAA43-R-XbaI, and cloned into $S a c \mathrm{I}$ and $X b a \mathrm{I}$ digested pDHC29. Both constructs were then confirmed by DNA sequencing.

Culture medium

M9 minimal medium contains $6.78 \mathrm{~g} / \mathrm{L}$ of $\mathrm{Na}_{2} \mathrm{HPO}_{4}$, $0.5 \mathrm{~g} / \mathrm{L}$ of $\mathrm{NaCl}, 1 \mathrm{~g} / \mathrm{L}$ of $\mathrm{NH}_{4} \mathrm{Cl}$, and $3 \mathrm{~g} / \mathrm{L}$ of $\mathrm{KH}_{2} \mathrm{PO}_{4}$ supplemented with $0.2 \%$ of glucose and $1 \mathrm{mM}$ of $\mathrm{MgSO}_{4}$. Buffered LB medium is Luria-Bertani (LB) broth supplemented with $0.1 \mathrm{M}$ sodium phosphate buffer.

\section{2-haloacrylate reductase (CAA43) assay}

Test strains were first grown in $5 \mathrm{~mL}$ of LB broth overnight and supplemented with antibiotics such as $100 \mu \mathrm{g} / \mathrm{mL}$ of ampicillin or $35 \mu \mathrm{g} / \mathrm{mL}$ of chloramphenicol if required. Overnight culture $(1.5 \mathrm{~mL})$ was then inoculated to $150 \mathrm{~mL}$ of fresh LB broth in $500 \mathrm{~mL}$ flasks supplemented with antibiotics and grown in a rotary shaker at $250 \mathrm{rpm}$ and $37{ }^{\circ} \mathrm{C}$. After $3-4 \mathrm{~h}$ growth, IPTG was added to make the concentration $1 \mathrm{mM}$ in each flask to induce expression of CAA43 from pTrc99A-CAA43. In the case with pASKIBA3-G6PD1, $0.2 \mathrm{mg} / \mathrm{mL}$ of anhydrotetracycline was added to induce expression of G6PDH. The induction continued under the same conditions for $18 \mathrm{~h}$ before cells were collected for the subsequent experiments.

After $18 \mathrm{~h}$ of induction, equal amount of cells were collected based on optical density measurements at OD $600 \mathrm{~nm}$. Cell pellets were then washed with minimal medium without nitrogen source and resuspended in $15 \mathrm{~mL}$ of reaction medium. Minimal medium without nitrogen source contains $6 \mathrm{~g} / \mathrm{L} \mathrm{Na}_{2} \mathrm{HPO}_{4}, 3 \mathrm{~g} / \mathrm{L} \mathrm{KH}_{2} \mathrm{PO}_{4}$, and $0.5 \mathrm{~g} / \mathrm{L} \mathrm{NaCl}$. Reaction medium is minimal medium without nitrogen source supplemented with $0.1 \mathrm{mM} \mathrm{CaCl}_{2}, 1 \mathrm{mM} \mathrm{MgSO}_{4}$, $1 \mathrm{mg} / \mathrm{mL}$ thiamine, $2 \%$ glucose, $50 \mathrm{mM}$ 2-chloroacrylic acid, and antibiotics when appropriate. $\mathrm{pH}$ of the reaction medium was adjusted to 7.0 with $6 \mathrm{~N} \mathrm{HCl}$. 
Table 1 Strains and plasmids

\begin{tabular}{|c|c|c|}
\hline & Genotype & Reference \\
\hline \multicolumn{3}{|l|}{ Strains } \\
\hline MQ & 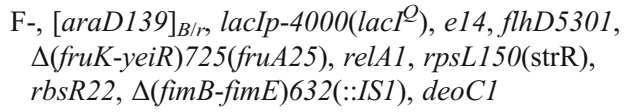 & From CGSC, (Lovingshimer et al. 2006) \\
\hline RL257 & MQ pfkA203(del-ins)::FRT, pfkB205(del-ins)::FRT & From CGSC, (Lovingshimer et al. 2006) \\
\hline RL254 & MQ pfkA203(del-ins)::FRT & From CGSC, (Lovingshimer et al. 2006) \\
\hline RL255 & MQ $p f k B 205$ (del-ins)::FRT & From CGSC, (Lovingshimer et al. 2006) \\
\hline YW8055 & MQ pACLYC & This study \\
\hline YW8056 & RL257 pACLYC & This study \\
\hline YW8057 & RL254 pACLYC & This study \\
\hline YW8058 & RL255 pACLYC & This study \\
\hline YW8082 & MQ pACLYC pASK-IBA3-G6PD1 & This study \\
\hline YW8083 & RL257 pACLYC pASK-IBA3-G6PD1 & This study \\
\hline YW8084 & RL254 pACLYC pASK-IBA3-G6PD1 & This study \\
\hline YW8085 & RL255 pACLYC pASK-IBA3-G6PD1 & This study \\
\hline YW8051 & MQ pTrc99A-CAA43 & This study \\
\hline YW8052 & RL257 pTrc99A-CAA43 & This study \\
\hline YW8053 & RL254 pTrc99A-CAA43 & This study \\
\hline YW8054 & RL255 pTrc99A-CAA43 & This study \\
\hline YW8066 & MQ pDHC29-CAA43 & This study \\
\hline YW8067 & RL257 pDHC29-CAA43 & This study \\
\hline YW8068 & RL254 pDHC29-CAA43 & This study \\
\hline YW8069 & RL255 pDHC29-CAA43 & This study \\
\hline YW8070 & MQ pDHC29-CAA43 pASK-IBA3-G6PD1 & This study \\
\hline YW8071 & RL257 pDHC29-CAA43 pASK-IBA3-G6PD1 & This study \\
\hline YW8072 & RL254 pDHC29-CAA43 pASK-IBA3-G6PD1 & This study \\
\hline YW8073 & RL255 pDHC29-CAA43 pASK-IBA3-G6PD1 & This study \\
\hline \multicolumn{3}{|l|}{ Plasmids } \\
\hline pET101-D-topo-CAA43 & $\begin{array}{l}\text { CAA43, encoding 2-haloacrylate reductase from } \\
\text { Burkholderia sp. WS, was expressed in } \\
\text { pET101/D-TOPO (Invitrogen), } \text { Ap }^{\mathrm{R}}\end{array}$ & (Kurata et al. 2005), (Kurata et al. 2008) \\
\hline pTrc99A-CAA43 & CAA43 expressed in $p \operatorname{Trc} 99 A, A p^{R}$ & This study \\
\hline pDHC29 & High copy plasmid compatible with pASK-IBA3 & (Phillips et al. 2000) \\
\hline pDHC29-CAA43 & CAA43 expressed in $\mathrm{pDHC} 29, \mathrm{Cm}^{\mathrm{R}}$ & This study \\
\hline pACLYC & $\begin{array}{l}\text { crtE, crtI, and } c r t B \text { from Erwinia herbicola cloned } \\
\text { in pACYC184 }\end{array}$ & (Cunningham et al. 1994) \\
\hline pASK-IBA3-G6PD1 & $\begin{array}{l}\text { P1 isoform of Arabidopsis glucose-6-phosphate } \\
\text { dehydrogenase AtG6PD1 cloned in pASK-IBA3 } \\
\text { (IBA, St. Louis, MO, USA), Ap }{ }^{\mathrm{R}}\end{array}$ & (Wakao and Benning 2005) \\
\hline
\end{tabular}

After cells were resuspended in reaction medium, around $14.5 \mathrm{~mL}$ aliquots of the suspension of each strain were distributed into $15 \mathrm{~mL}$ glass vials. The glass vials were later closed and sealed with rubber stoppers for anaerobic fermentation. Samples were taken using sterile needle and syringe at each time point for HPLC analysis.

\section{Lycopene assay}

Test strains were first grown in $5 \mathrm{~mL}$ of LB broth overnight at $37{ }^{\circ} \mathrm{C}$. Then $500 \mu \mathrm{L}$ of the overnight cultures were inoculated into $50 \mathrm{~mL}$ of fresh LB supplemented with $2 \%$ glucose, $100 \mathrm{mM}$ phosphate buffer (pH7.0) and antibiotics, when appropriate, and the cultures were grown in $250 \mathrm{~mL}$ flasks in a rotary shaker at $30^{\circ} \mathrm{C}, 250 \mathrm{rpm}$. After 24 or $48 \mathrm{~h}$, $10 \mathrm{~mL}$ samples from each flask were taken, the cells were pelleted and washed once with distilled water. The cell pellets were then resuspended in $5 \mathrm{~mL}$ of acetone at $55{ }^{\circ} \mathrm{C}$ for $15 \mathrm{~min}$ in the dark to extract lycopene from the cells. After a 15-min extraction, the solutions were centrifuged at $11,000 \mathrm{rpm}$ for $10 \mathrm{~min}$ to remove cell debris (Martinez et al. 2008). The supernatants were then measured for absorbance at 
OD475nm for lycopene concentration. A calibration curve was made using lycopene standard (Sigma, St. Louis, MO). The extinction coefficient used was $3.82 \mathrm{~cm}^{-1} \mathrm{~L} / \mathrm{mg}$.

\section{Metabolite analysis by HPLC}

Samples of $600 \mu \mathrm{L}$ at each time point were taken and centrifuged at 12,000 rpm for $1 \mathrm{~min}$. The supernatants were then filtered through a $0.2-\mu \mathrm{m}$ syringe filter and aliquots were subjected to HPLC analysis. To measure extracellular metabolites, CAA43 substrate, 2- chloroacrylic acid and product, 2-chloropropionic acid, a HPLC system from Shimadzu Scientific Instruments was used. The metabolites were separated by a cation-exchange column (HPX-87H, BioRad Labs, CA) and quantified by a differential refractive index detector (Waters 2410, Milford, MA) and a UV-VIS detector (SPD-10A, Shimadzu Scientific Instruments, Columbia, MD). The mobile phase was $2.5 \mathrm{mM}$ sulfuric acid and column was operated with a flow rate of $0.5 \mathrm{~mL} / \mathrm{min}$ at a constant temperature of $55^{\circ} \mathrm{C}$.

\section{Results}

To test that deletion of both PFK isoenzymes in E. coli will increase NADPH bioavailability, we obtained $p f k A p f k B$ double null strain RL257 from the E. coli Genetic Stock Center along with parent strain $\mathrm{MQ}$, and the $p f k A$ single deletion strain, RL254, and the $p f k B$ single deletion strain, RL255. These four isogenic strains were confirmed by growing in M9 minimal medium with glucose as the sole carbon source. Consistent with the previous study (Lovingshimer et al. 2006), MQ and the $p f k B$ deletion strain, RL255 grew well in minimal medium with $0.2 \%$ glucose as carbon source, while the $p f k A$ pfkB double-deletion strain, RL257 and the $p f k A$ deletion strain, RL254 showed no or little growth. This result is consistent with the fact that PfkA contributes the majority of the PFK activity while PfkB serves as the minor isoenzyme. Only when the major PFK $p f k A$ gene was deleted did we see a decreased growth.

To test the effect of the deletion of PFK activity on NADPH-dependent biosynthesis, we first tested the deletion strains on their ability to produce lycopene, which requires 16 mol of NADPH for $1 \mathrm{~mol}$ of product (Alper et al. 2005a). pACLYC, carrying the $c r t E$, $c r t B$, and $c r t I$, lycopene production genes from E. herbicola was transformed into MQ, RL257, RL254, and RL255, which resulted in testing strains YW8055, 8056, 8057, and 8058, respectively. The strains were grown in buffered LB medium with addition of $2 \%$ glucose for 24 to $48 \mathrm{~h}$, lycopene was then extracted by acetone and measured (Fig. 2) (Martinez et al. 2008). Results showed that wild-type YW8055 consistently produced less lycopene at $633 \mu \mathrm{g} / \mathrm{L}$ than the PFK null strain ( $p f k A p f k B)$ YW8056 at $1,055 \mu \mathrm{g} / \mathrm{L}$. The $p f k A$ single deletion strain, YW8057, showed the highest concentration of lycopene at $4,330 \mu \mathrm{g} / \mathrm{L}$, while the $p f k B$ single deletion, YW8058 had a concentration of lycopene intermediate between that of the double-deletion strain, YW8056 and the wild-type strain YW8055. The amount of lycopene observed per glucose consumed (Fig. 2b) indicated this value was highest for cultures of the $p f k A$ mutant strain followed by cultures of the double-deletion strain. This trend stayed true when lycopene yields were compared based on dry cell weight (DCW) (Fig. 2c).

In addition, we tested the combined effect of overexpression of G6PDH and PFK deletion on NADPH bioavailability. pASK-IBA3-G6PD1 carrying Arabidopsis G6PDH along with the lycopene production plasmid, pACLYC were transformed into MQ, RL257, RL254, and RL255 resulting in testing strains YW8082, YW8083, YW8084, and YW8085, respectively. These strains were grown in buffered LB medium supplemented with $2 \%$ glucose, ampicillin $100 \mu \mathrm{g} / \mathrm{mL}$, and chloremphenicol $5 \mu \mathrm{g} / \mathrm{mL}$ for 24 to $66 \mathrm{~h}$. At the end of the culture, cells were collected and extracted for lycopene extraction. As shown in Fig. 3, after $66 \mathrm{~h}$ of growth, the doubledeletion strain, $p f k A p f k B$, produced the highest yield at $37 \mathrm{mg}$ lycopene/mole of glucose consumed, while the $p f k B$ single deletion strain produced the second highest yield at $24 \mathrm{mg}$ of lycopene/mole of glucose consumed, and the parent control MQ and $p f k A$ single deletion strain produced comparable and the lowest yields of lycopene at 10 and $13 \mathrm{mg}$ lycopene/mole of glucose consumed, respectively. Compared to the lycopene/ cell amount or glucose consumed of the PFK deletion only strains, additional overexpression of G6PDH did not increase lycopene production. The same trend was also observed when lycopene yields were compared based on DCW as shown in Fig. 3c.

Since we are re-routing the pathway glucose being oxidized to PPP, to avoid the complexity caused by the precursors supplied by glycolytic pathway such as production of lycopene, we examined a simplified system representative of a whole cell biocatalyst process. We also wanted to use a situation where we could closely quantify the amount of glucose consumed and the amount of reductant consumed in the formation of the NADPH-requiring product compared to the total amount of reductant generated by the cell. In this experiment, we used anaerobic conditions so we could assess the proportion of total reduced metabolism that proceeded through the added heterologous NADPH consumption system. This approach employed a one-step reaction system using 2-haloacrylate reductase CAA43 which was added to the PFK deletion strains. CAA43 from bacteria Burkholderia sp. WS catalyzes a single-step reaction that reduces 2-chloroacrylate to 2-chloropropionic acid using NADPH as cofactor. CAA43 was first cloned in pTrc99A under the Ptrc promoter, resulting in pTrc99A-CAA43. This plasmid was then transformed into MQ, RL257, RL254, and RL255, resulting in testing strains YW8051, 8052, 8053, and 8054, respectively. These strains were first grown in LB 
Fig. 2 Lycopene production in PFK-deficient strains. a Lycopene concentration of wild-type MQ and PFK single or double-deletion strains. Samples were taken after 26, 48 , and $76 \mathrm{~h}$ growth in LB supplemented with $2 \%$ of glucose under $30^{\circ} \mathrm{C}$ and $250 \mathrm{rpm}$. b Lycopene production of wild-type MQ and PFK single or doubledeletion strains based on glucose consumption. Samples were taken after 26,48 , and $76 \mathrm{~h}$ growth in LB supplemented with $2 \%$ of glucose under $30^{\circ} \mathrm{C}$ and 250 rpm. c Lycopene production (microgram) based on dry cell weight $(\mathrm{DCW})(\mathrm{g})$. $1 \mathrm{OD}_{600 \mathrm{~nm}}=0.33 \mathrm{~g} \mathrm{DCW} / \mathrm{L}$ a
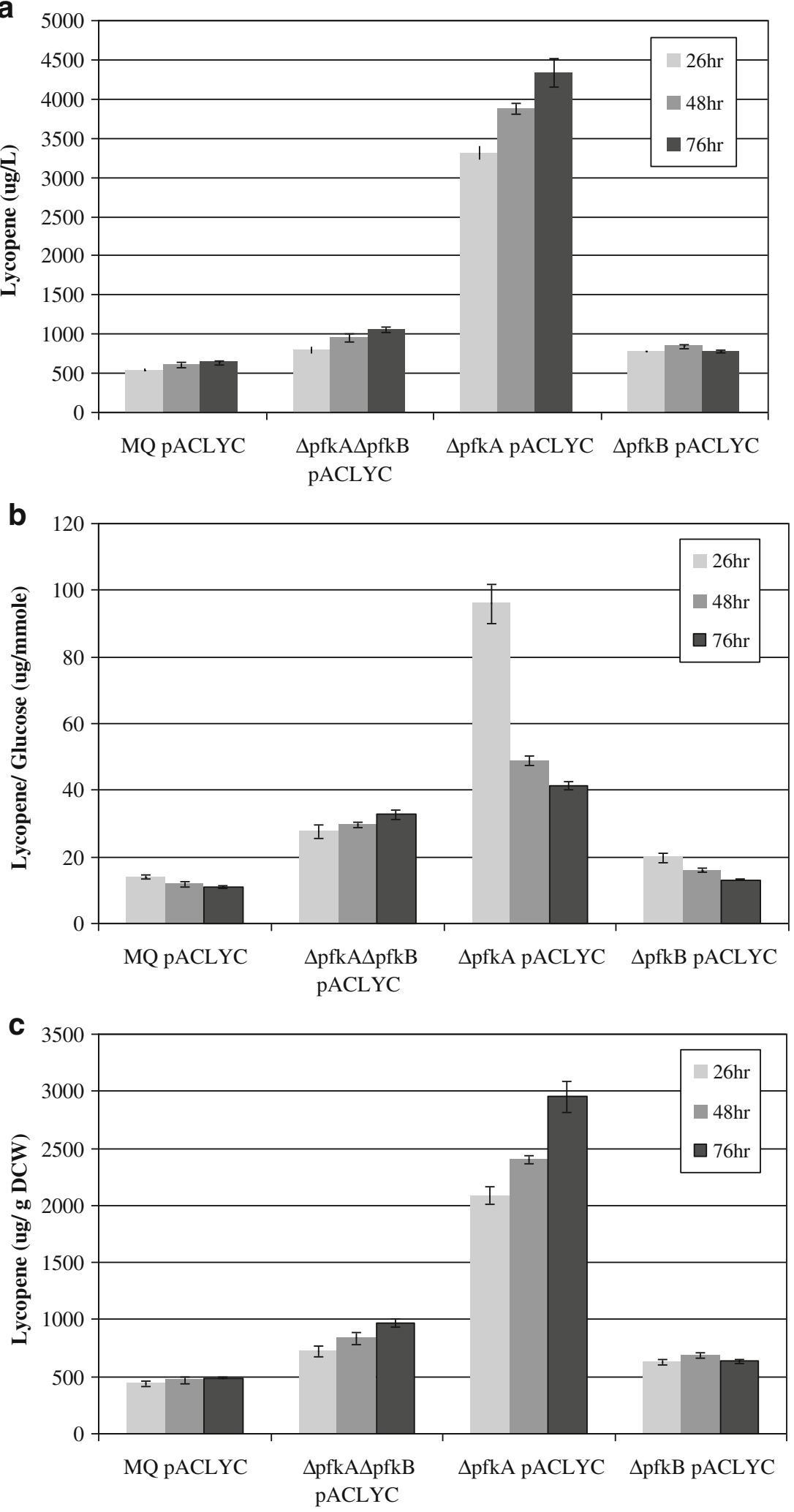

medium, and CAA43 expression was induced by IPTG. The cells were collected and resuspended in non-growing minimal salts without nitrogen. The substrate of the one-step reaction, 2-chloroacrylate was added at the same time at a concentration of $50 \mathrm{mM}$, and the reactions were carried out with the resuspended non-growing cells in a $37{ }^{\circ} \mathrm{C}$ shaker. As shown in Fig. 4, the double-deletion strain, YW8052 showed the highest yield of 2-chloropropionic acid of 0.92 
Fig. 3 Combined effects of G6PDH overexpression and PFK deficiency on lycopene production. a Lycopene (microgram per liter) produced by PFK-deficient strains (YW8055, 8056, 8057, and 8058) and PFK-deficient strains with coexpressed G6PDH (YW8082, 8083, 8084, and 8085). pG6PDH refers to pASK-IBA3-G6PD1. b Lycopene production based on glucose consumption for PFKdeficient strains and PFKdeficient strains with overexpression of G6PDH. c Lycopene production (microgram) based on dry cell weight (DCW) (g).

$1 \mathrm{OD}_{600 \mathrm{~nm}}=0.33 \mathrm{~g} \mathrm{DCW} / \mathrm{L}$
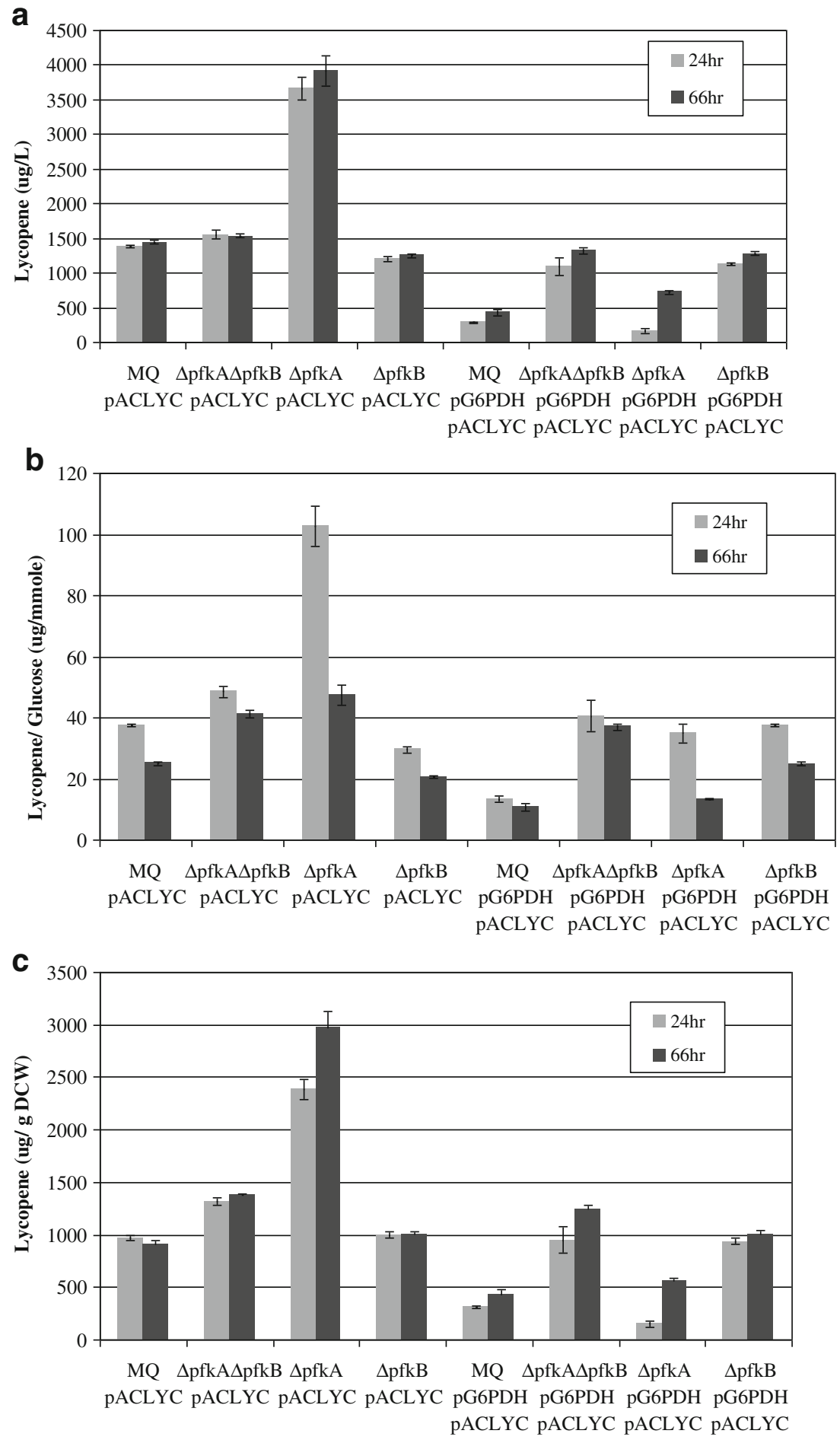

product/glucose $(\mathrm{M} / \mathrm{M})$, while the $p f k A$ single deletion strain, YW8053 had the second highest yield of 0.34 product/glucose $(\mathrm{M} / \mathrm{M})$, and the $p f k B$ single deletion strain, YW8054 and parent control strain YW8051 showed comparable yields of 0.22 and 0.19 product/glucose $(\mathrm{M} / \mathrm{M})$, respectively. In Fig. 4b, the proportion of reduction equivalents that go toward formation of the reduced 2-chloropropionic acid compared to the total reduced metabolites is calculated and presented to give a picture of how much of the total reductant available is routed through the added NADPH-requiring pathway. In the 6-h determination, we see around $45 \%$ of total reductant in the double-deletion mutant strain goes to the reduction of the 2-chloroacrylate. The $p f k A$ mutant is the next most efficient with around $18 \%$, still significantly more than in the wild type ( 11\%). 
Fig. 4 a Production of 2chloropropionic acid based on glucose consumption. Cells of PFK-deficient strains transformed with pTrc99ACAA43 were collected and used as whole cell catalyst for one-step reduction of 2chloroacrylate. The yield was calculated as the amount of product 2-chloropropionic acid per mole of glucose consumed. b Percentage of reducing power in the form of NADPH among the total reducing power generated by the PFK-deficient cells. It was calculated as the amount of 2-chloropropionic acid generated vs. all the reduced metabolites resulting from the reduction reactions by $\mathrm{NADPH}$ and NADH, such as lactate, ethanol, succinate, and 2-chloropropionic acid

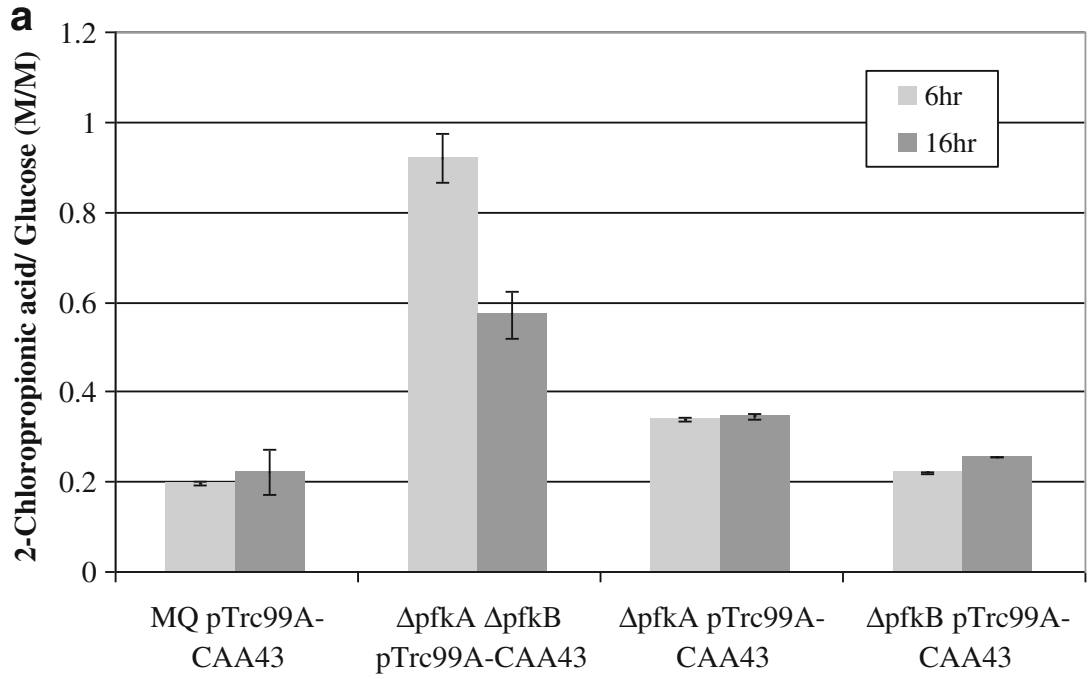

b

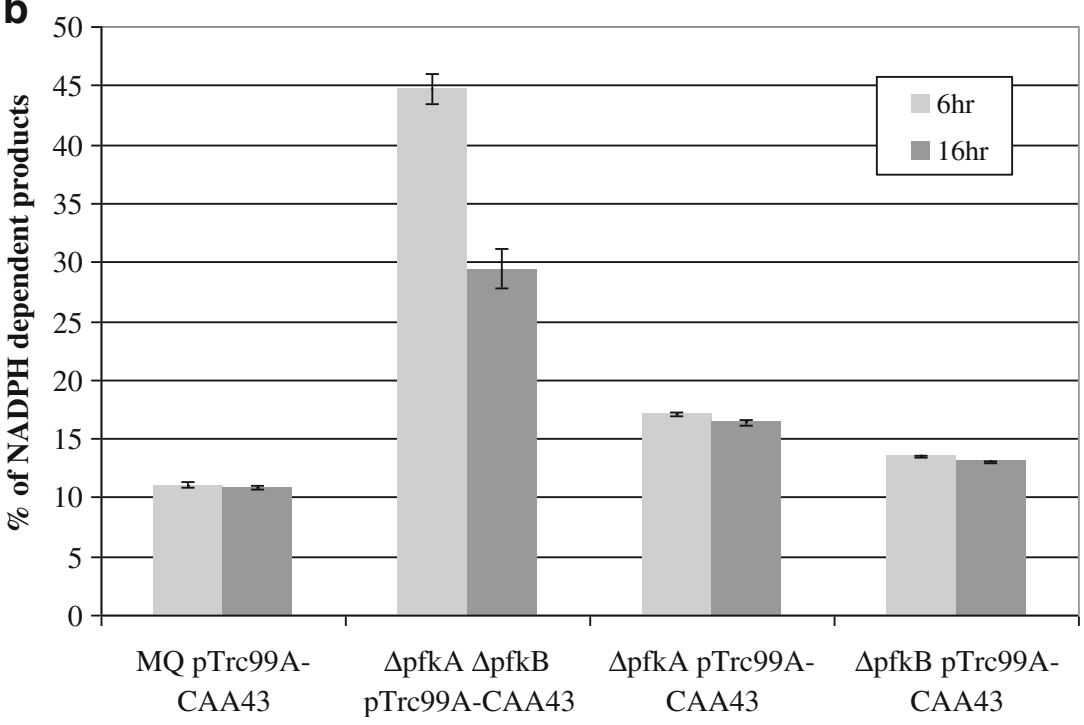

To test whether overexpression of G6PDH will enhance PPP and further increase the NADPH availability, we transformed the plasmid expressing G6PDH from Arabidopsis together with CAA43 into the set of PFK deletion strains. To be compatible with the G6PDH plasmid, pASK-IBA3-G6PD1, the gene encoding CAA43 was transferred to pDHC29. The testing strains were therefore YW8066, 8067, 8068, 8069, 8070, 8071, 8072, and 8073 (Table 1). They were first grown in LB medium and G6PDH was induced with anhydrotetracycline, and CAA43 was induced with IPTG. As seen from Fig. 5, overexpression of G6PDH indeed increased the yield calculated as product/glucose for the double-deletion strain, the $p f k A$ single deletion, and the $p f k B$ single deletion strain. The most pronounced increase was with the double-deletion PFK strain, while the parental control showed a little lower yield compared to that found without G6PDH overexpression.

Analyzing the metabolite profiles for this experiment, we noticed that large amounts of lactate were produced as byproduct, since we set up the CAA43 assay under anaerobic conditions in order to be able to quantify the sources and products of reduction reactions. To reduce the carbon and reductant waste, we introduced a lactate dehydrogenase deletion (ldh::kan) into the testing strains. However, after $l d h$ was deleted, the testing strains stopped using glucose (data not shown). We speculate this is because without a healthy outlet for NADH oxidation, the disruption of the NADH/NAD ${ }^{+}$ balance could not be entirely replaced by the NADPH sink and caused the cells to stop glucose uptake.

\section{Discussion}

Regeneration of reduced cofactor has been an important factor in enzyme based bioconversions such as reduction of a carbonyl group to a chiral alcohol, which are often important intermediates for pharmaceutical compounds. Often such reductions employ NADPH, which is in short supply in cells and can be a limiting factor in formation of 
Fig. 5 Combined effects of G6PDH overexpression and PFK deficiency based on 2haloacrylate reductase CAA43 assay. a Yield of 2-

chloropropionic acid based on glucose consumption, produced by PFK-deficient strains (YW8066, 8067, 8068, and 8069) and PFK-deficient strains with G6PDH overexpressed (YW8070, 8071, 8072, and 8073). pG6PDH is pASKIBA3-G6PD1, and pCAA43 refers to pDHC29-CAA43. b Percentage of reducing power in the form of NADPH among the total reducing power generated by the PFK-deficient cells with or without G6PDH overexpression. It was calculated as the amount of 2chloropropionic acid generated vs. all the reduced metabolites resulting from the reduction reactions by both NADH and $\mathrm{NADPH}$, such as lactate, ethanol, succinate, and 2chloropropionic acid
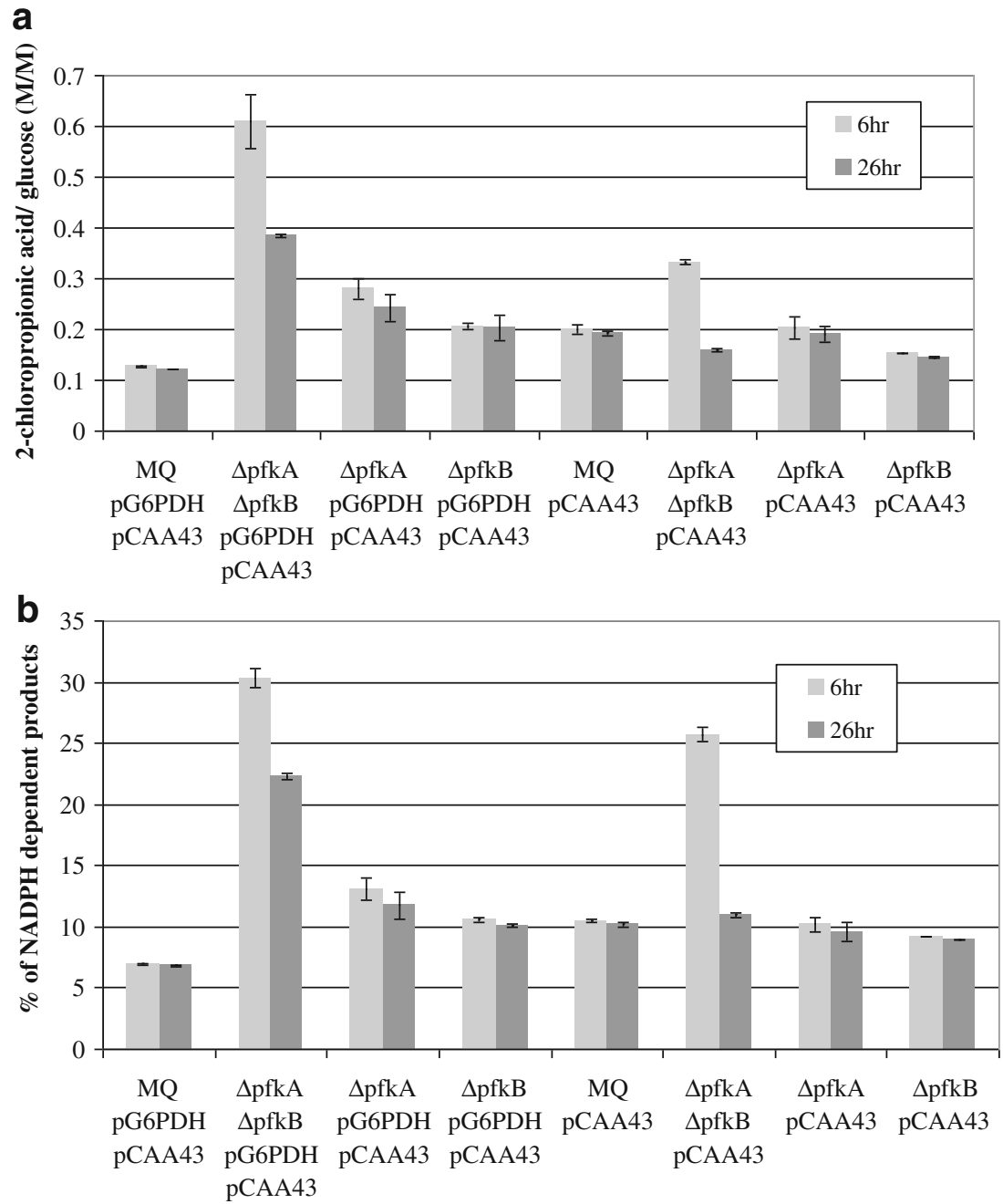

high yields. This limitation has been addressed on a reaction level by protein engineering of the enzyme from a NADPHrequiring one to an NADH-utilizing enzyme. Other efforts to supply NADPH at an enhanced level have used strategies at the cellular level, including replacement of a NADHdependent reaction of glycolysis by a NADPH-dependent one, rerouting more flux through the NADPH-generating pentose phosphate pathway, or employing or removing transhydrogenases. In this study, we focused on NADPH generation through the pentose phosphate pathway (PPP). By deleting the both PFK genes $p f k A$ and $p f k B$ in E. coli, the glycolysis pathway was interrupted, while glucose was forced to mainly go through the PPP to be oxidized, therefore more reducing equivalents in the form of NADPH should be produced and be available for formation of reduced compounds requiring this specific cofactor.

Recent work has been published in examination of this strategy but with somewhat different background strains and using various compounds and pathways to evaluate the effects on NADPH availability. NADPH-dependent bioconversions were studies by Walton and Stewart (2004), using either a NADPH-dependent monooxygenase reaction or a NADPH-dependent conversion of ethyl acetoacetate in nongrowing aerobic cells where yields of $2.3 \mathrm{~mol}$ of reduced product per mole of glucose consumed were observed in the latter case. Chin and Cirino (2011) examined combinations of $p f k$ mutations and transhydrogenase mutants on the production of xylitol and aerobic experiments gave a high yield of $5.4 \mathrm{~mol} / \mathrm{mol}$ glucose with a sthA pfkA mutant. In other recently reported studies, the reduction of methyl acetoacetate by an alcohol dehydrogenase was analyzed in whole cell biotransformations. The yield in a pgi mutant increased to 3.78 vs. 2.44 in the parent strain, and higher yields of 4.79 or 5.46 were reported in cells lacking phosphofructokinase I or I and II, respectively (Siedler et al. 2011). This work was followed by additional mutant studies where the authors confirmed by ${ }^{13} \mathrm{C}$ metabolic flux analysis that partial cyclization of the pentose phosphate pathway occurred, as we considered in the experiments here. They also further extended the work and found a higher glucose uptake rate in each of the host strains examined when a glucose facilitator and glucose kinase system were added to the strains. In this case, the strains were 
based on the parent, E. coli BL21 Star (DE3) and pgi, pfkA, $p f k A$ pfkB derivatives were studied. To explain the differences in the values seen in the experiments by other workers and in this report, the different background hosts and the different conditions examined are noted as well as the specific experimental objectives of each report. The work we report is consistent with these reports of increased flow through the pentose phosphate pathway generating more availability of NADPH.

Specifically in our tests of $p f k$ mutants, we found unexpectedly that the $p f k A$ single deletion strain produced more lycopene and demonstrated a higher yield. In some cases cited above, the double mutant was slightly improved over the $p f k A$ mutant; however, in those cases, the product was not coupled to the formation of glycolytic intermediates. Based on a previous study, except for NADPH, lycopene production was also limited by its precursor, glyceraldehyde 3-phosphate (Farmer and Liao 2001). In the wild-type $E$. coli, $1 \mathrm{~mol}$ of glucose generates $2 \mathrm{~mol}$ of glyceraldehyde 3-phosphate, while in a PFK null strain, 1 mol of glucose would be expected to generate $1 \mathrm{~mol}$ of glyceraldehyde 3-phosphate while up to 3 mol of carbon are lost in the form of $\mathrm{CO}_{2}$. For this reason, we speculate that even with extra NADPH, the PFK null strain, YW8055 will not be able to produce more lycopene due to limitations of the precursor, glyceraldehyde 3-phosphate and its subsequent derived product pyruvate which is also a substrate of DXP synthase. The effects of low levels of these precursors and competition from other enzymes in the cell for these substrates make postulating precise reasons for the effect difficult. Some general thoughts are presented here on the relative importance of various factors. In the case of the $p f k A$ single deletion strain, the remaining PfkB can convert part of the fructose-6-phosphate to fructose-1,6-biphosphate and allow some flux down glycolysis to generate glyceraldehyde-3phosphate. At the same time, the rest of the fructose-6phosphate can be passed through the PPP and generate NADPH. The flux distribution of fructose-6-phosphate in $p f k A$ single deletion strain was not measured in this study so how much fructose-6-phosphate goes through glycolysis through PfkB or how much goes through the PPP was not determined. But the fact that the $p f k A$ single deletion strain showed the highest yield of lycopene lead us to consider the possibility that in this particular strain the carbon flux distribution is more preferable for lycopene production with a balanced ratio of precursor vs. NADPH required.

Glucose-6-phosphate dehydrogenase is the first enzyme when glucose-6-phosphate enters the PPP, which converts glucose-6-phosphate to 6-phosphoglucono- $\delta$ - lactone with $\mathrm{NADP}^{+}$as the electron acceptor. By overexpressing
G6PDH, the metabolic flux that goes to the PPP would be expected to increase and NADPH production would therefore also be increased. E. coli G6PDH is regulated by NADH level in the cell, so to avoid the inhibition, we chose one isoform of G6PDH from Arabidopsis, G6PD1, which is reported to be insensitive to redox changes (Wakao and Benning 2005). However, we found further enhancement of PPP by overexpressing Arabidopsis G6PDH did not further increase the lycopene yield. Since lycopene production may have also limited by precursor glyceraldehyde-3phosphate and pyruvate, this result may reflect a more complicated metabolic situation.

To avoid the complication arising from use of the precursors from glycolysis in the analyzed product formation, we developed a one-step reaction system utilizing CAA43 in order to have a better assessment of the NADPH bioavailability. The CAA43 catalyzed reaction reduces $1 \mathrm{~mol}$ of 2-chloroacylic acid to $1 \mathrm{~mol}$ of 2-chloropropionic acid coupled with oxidation of $1 \mathrm{~mol}$ of NADPH to NADP ${ }^{+}$ and neither of these compounds is normally produced or acted upon by $E$. coli, we would be able to separate the use of NADPH by this sink without direct effects on the usual metabolites of the cell. The advantage of the single-step reaction is that it directly reflects the bioavailability of $\mathrm{NADPH}$, and the biocatalysis was carried out under anaerobic conditions which allow us to quantify and calculate the percentage of the reducing power in the form of NADPH (the relative amount going to 2-chloropropionic acid) vs. NADH (as measured by the amount of products arising from NADH-dependent formation of lactate as the major product and to a lesser extent the small quantities of ethanol and succinate). The results shown here are consistent with our view and previous work (Chin and Cirino 2011) that deletion of PFK would increase the NADPH availability, especially when considering on a per glucose basis. For all the reducing power generated by the cells, the PFK deletion strains have more in the form of NAPDH. The results suggests to us that modulation of the $p f k$ genes can be used in applications to improve production of NADPH-dependent chemicals and that fine tuning of this node along with consideration of transhydrogenase contributions, and glucose uptake rates can help in the design of processes for production of compounds requiring NADPH reductive steps.

Acknowledgement This work was supported in part by National Science Foundation CBET0828516. Y. Wang was partially supported by a postdoctoral fellowship from the HHMI Beyond Traditional Borders program and by a John S. Dunn Foundation Collaborative Research Award. The authors want to thank Prof. Kurata for providing plasmid pET101-D-topo-CAA43 and Prof. Benning for the gift of pASK-IBA3-G6PD1. 


\section{References}

Alper H, Jin YS, Moxley JF, Stephanopoulos G (2005a) Identifying gene targets for the metabolic engineering of lycopene biosynthesis in Escherichia coli. Metab Eng 7(3):155-164. doi:10.1016/ j.ymben.2004.12.003

Alper H, Miyaoku K, Stephanopoulos G (2005b) Construction of lycopene-overproducing $E$. coli strains by combining systematic and combinatorial gene knockout targets. Nat Biotechnol 23(5):612-616. doi:10.1038/nbt1083

Bartek T, Blombach B, Zonnchen E, Makus P, Lang S, Eikmanns BJ, Oldiges M (2010) Importance of NADPH supply for improved Lvaline formation in Corynebacterium glutamicum. Biotechnol Prog 26(2):361-371. doi:10.1002/btpr.345

Chemler JA, Fowler ZL, McHugh KP, Koffas MA (2010) Improving NADPH availability for natural product biosynthesis in Escherichia coli by metabolic engineering. Metab Eng 12(2):96-104. doi:10.1016/j.ymben.2009.07.003

Chin JW, Cirino PC (2011) Improved NADPH supply for xylitol production by engineered Escherichia coli with glycolytic mutations. Biotechnol Prog 27(2):333-341. doi:10.1002/btpr.559

Chin JW, Khankal R, Monroe CA, Maranas CD, Cirino PC (2009) Analysis of NADPH supply during xylitol production by engineered Escherichia coli. Biotechnol Bioeng 102(1):209220. doi:10.1002/bit.22060

Cunningham FX, Jr., Sun Z, Chamovitz D, Hirschberg J, Gantt E (1994) Molecular structure and enzymatic function of lycopene cyclase from the cyanobacterium Synechococcus sp strain PCC7942. Plant Cell 6(8):1107-1121. doi:10.1105/tpc.6.8.1107

Farmer WR, Liao JC (2001) Precursor balancing for metabolic engineering of lycopene production in Escherichia coli. Biotechnol Prog 17(1):57-61. doi:10.1021/bp000137t

Kim S, Lee CH, Nam SW, Kim P (2011a) Alteration of reducing powers in an isogenic phosphoglucose isomerase (pgi)-disrupted Escherichia coli expressing NAD(P)-dependent malic enzymes and NADPdependent glyceraldehyde 3-phosphate dehydrogenase. Lett Appl Microbiol 52(5):433-440. doi:10.1111/j.1472-765X.2011.03013.x

Kim YM, Cho HS, Jung GY, Park JM (2011b) Engineering the pentose phosphate pathway to improve hydrogen yield in recombinant Escherichia coli. Biotechnol Bioeng 108(12):2941-2946. doi:10.1002/bit.23259

Kurata A, Kurihara T, Kamachi H, Esaki N (2005) 2-Haloacrylate reductase, a novel enzyme of the medium chain dehydrogenase/ reductase superfamily that catalyzes the reduction of a carboncarbon double bond of unsaturated organohalogen compounds. J Biol Chem 280(21):20286-20291. doi:10.1074/jbc.M414605200

Kurata A, Fujita M, Mowafy AM, Kamachi H, Kurihara T, Esaki N (2008) Production of (S)-2-chloropropionate by asymmetric re- duction of 2-chloroacrylate with 2-haloacrylate reductase coupled with glucose dehydrogenase. J Biosci Bioeng 105(4):429-431. doi:10.1263/jbb.105.429

Lee WH, Park JB, Park K, Kim MD, Seo JH (2007) Enhanced production of epsilon-caprolactone by overexpression of NADPHregenerating glucose 6-phosphate dehydrogenase in recombinant Escherichia coli harboring cyclohexanone monooxygenase gene. Appl Microbiol Biotechnol 76(2):329-338. doi:10.1007/s00253007-1016-7

Lovingshimer MR, Siegele D, Reinhart GD (2006) Construction of an inducible, $p f k A$ and $p f k B$ deficient strain of Escherichia coli for the expression and purification of phosphofructokinase from bacterial sources. Protein Expr Purif 46(2):475-482. doi:10.1016/ j.pep.2005.09.015

Martinez I, Zhu J, Lin H, Bennett GN, San KY (2008) Replacing Escherichia coli NAD-dependent glyceraldehyde 3-phosphate dehydrogenase (GAPDH) with a NADP-dependent enzyme from Clostridium acetobutylicum facilitates NADPH dependent pathways. Metab Eng 10(6):352-359. doi:10.1016/j.ymben.2008. 09.001

Phillips GJ, Park SK, Huber D (2000) High copy number plasmids compatible with commonly used cloning vectors. Biotechniques 28(3):400-402, 404, 406 passim

Sanchez AM, Andrews J, Hussein I, Bennett GN, San KY (2006) Effect of overexpression of a soluble pyridine nucleotide transhydrogenase (UdhA) on the production of poly(3-hydroxybutyrate) in Escherichia coli. Biotechnol Prog 22(2):420-425. doi:10.1021/ bp050375u

Siedler S, Bringer S, Bott M (2011) Increased NADPH availability in Escherichia coli: improvement of the product per glucose ratio in reductive whole-cell biotransformation. Appl Microbiol Biotechnol 92(5):929-937. doi:10.1007/s00253-011-3374-4

Siedler S, Bringer S, Blank LM, Bott M (2012) Engineering yield and rate of reductive biotransformation in Escherichia coli by partial cyclization of the pentose phosphate pathway and PTS-independent glucose transport. Appl Microbiol Biotechnol 93(4):1459-1467. doi:10.1007/s00253-011-3626-3

Vinopal RT, Clifton D, Fraenkel DG (1975) PfkA locus of Escherichia coli. J Bacteriol 122(3):1162-1171

Wakao S, Benning C (2005) Genome-wide analysis of glucose-6phosphate dehydrogenases in Arabidopsis. Plant J 41(2):243256. doi:10.1111/j.1365-313X.2004.02293.x

Walton AZ, Stewart JD (2004) Understanding and improving NADPHdependent reactions by nongrowing Escherichia coli cells. Biotechnol Prog 20(2):403-411. doi:10.1021/bp030044m

Yoon KW, Doo EH, Kim SW, Park JB (2008) In situ recovery of lycopene during biosynthesis with recombinant Escherichia coli. J Biotechnol 135(3):291-294. doi:10.1016/j.jbiotec. 2008.04.001 